\title{
Analysis of Autoantibodies to Recombinant La (SS-B) Peptides in Systemic Lupus Erythematosus and Primary Sjogren's Syndrome
}

\author{
Paolo Bini, Jia-Li Chu, Charles Okolo, and Keith Elkon \\ The Hospital for Special Surgery, Cornell University Medical Center, New York, New York 10021
}

\begin{abstract}
Autoantibodies to a polymerase III transcription factor, La (SS-B), are frequently detected in the serum of patients with Sjogren's syndrome and systemic lupus erythematosus. To define the humoral immune response to this protein, we analyzed the patterns of antibody recognition toward 13 recombinant La peptides by immunoblotting and determined the heterogeneity of antibodies reactive with the immunodominant epitopes. The smallest epitopes that were strongly antigenic and recognized by $>70 \%$ of sera tested (immunodominant) were encoded by the subclones $\mathrm{BgX}$ and $\mathrm{XA}$ located in the $5^{\prime}$ and $3^{\prime}$ halves of the La cDNA, respectively. Conformation of the immunodominant La peptides played a major role in antibody recognition. Although greater diversity in antibody binding to carboxyl-terminal La peptides was observed, the overall pattern of peptide recognition by anti-La antibodies was similar in different diseases. The antibody responses to the immunodominant peptides were strongly correlated $(r=0.68, P<0.001)$. One- and two-dimensional isoelectric focusing of affinity purified IgG anti-La peptide antibodies revealed restricted heterogeneity and oligoclonal bands ( $\alpha$ light chains). These observations suggest that anti-La antibodies are induced and/or maintained by the self antigen and that their diversity is constrained either by mechanisms related to tolerance or by affinity maturation of the humoral immune response. (J. Clin. Invest. 1990. 85:325333.) autoantibody • epitope mapping • La (SS-B) • systemic lupus erythematosus
\end{abstract}

\section{Introduction}

Autoantibodies to intracellular proteins are markers of multisystem autoimmune diseases $(1,2)$. Several theories (e.g., polyclonal B cell activation, molecular mimicry, immune response to selected self antigens) have been proposed to account for autoantibody production (reviewed in reference 3 ). To evaluate these hypotheses, we previously analyzed the humoral immune response to a lupus autoantigen on ribosomal $P$ proteins. The similarities between immune recognition of the $P$ proteins and the properties described for foreign protein antigens suggested that anti-P antibodies were produced as an immune response to the autoantigens (4-6). Other investigators have demonstrated multiple epitopes on two different lupus autoantigens (8-10), supporting the notion that self pro-

Address reprint requests to Dr. Keith Elkon, The Hospital for Special Surgery, 535 East 70th Street, New York, NY 10021.

Received for publication $13 \mathrm{July} 1989$ and in revised form 3 October 1989.

J. Clin. Invest.

(C) The American Society for Clinical Investigation, Inc. 0021-9738/90/02/0325/09 \$2.00

Volume 85, February 1990, 325-333 teins drive autoantibody production. However, none of the latter studies characterized the properties of the epitopes, demonstrated that antibodies binding to each epitope are distinct, analyzed the heterogeneity of the autoantibodies reactive with the dominant epitopes, or compared epitope recognition in patients with different diseases. To address these questions, we evaluated the humoral immune response of clinically wellcharacterized patients with systemic lupus erythematosus (SLE), ${ }^{1}$ primary Sjogren's (sicca) syndrome (SS), and overlap syndromes to the autoantigen, La (SS-B). La is a 47-kD nuclear phosphoprotein $(11,12)$ that transiently binds RNA polymerase III transcripts of host and viral origin (13-15). La is required for efficient polymerase III transcription in vitro and functions as an auxiliary factor for termination of transcription (16). Anti-La (SS-B) antibodies are detected by immunodiffusion in $\sim 10 \%$ of patients with SLE, $50 \%$ of patients with SS, and a smaller number of patients with other autoimmune diseases (17-19). Antibodies against $\mathrm{La}$ and the associated protein, Ro (SS-A), are almost invariably found in mothers of infants with congenital heart block $(20,21)$.

\section{Methods}

Sera. Patients with SLE, SS, and overlap syndromes were selected for the study on the basis of a positive test for anti-La antibodies by counterimmunoelectrophoresis using a dog spleen extract as a source of antigen (13). 13 patients had SLE as defined by the revised American Rheumatism Association criteria (22), 11 patients had SS (keratoconjunctivitis sicca, xerostomia, and systemic features as described elsewhere [23]), and 6 patients had overlap syndromes (features of SLE combined with polymyositis and/or scleroderma).

La peptides. A cDNA obtained from a human Burkitt lymphoma library and encoding $87 \%$ of the total La protein was kindly provided by Dr. J. McNeilage (Walter and Eliza Hall Institute, Victoria, Australia) (7). The La cDNA and fragments obtained by digestion with restriction enzymes were subcloned into pATH plasmid expression vectors (kindly provided by $M$. Crivellone, Columbia University). One cDNA fragment, La SE (see Results), was subcloned into the pUEX 1 expression vector (24). La peptides were induced as tryptophanyl $E$ (trpE) fusion proteins (25) from the pATH plasmids in Escherichia coli strain RR1 with $50 \mu \mathrm{M}$ indoleacrylic acid. La SE was induced as a B-galactosidase fusion protein from the pUEX plasmid in $E$. coli strain MC 1061 by inactivating the thermolabile $\mathrm{CI}$ repressor at $42^{\circ} \mathrm{C}$. After induction, total protein lysates were made by boiling bacterial pellets in $1 \%$ SDS for 3 min.

Gel electrophoresis and immunoblotting. SDS-PAGE (26) and immunoblotting (27) were performed essentially as described elsewhere (13). Briefly, bacterial lysates (loaded at the same total protein concentration) were resolved on either $12 \%$ (trpE fusion proteins) or $8 \%$ (B-galactosidase-fusion protein) polyacrylamide gels and electrophoretically blotted to nitrocellulose paper. In some experiments duplicate nitrocellulose strips containing transferred proteins were autoclaved in

1. Abbreviations used in this paper: IEF, isoelectric focusing; SLE, systemic lupus erythematosus; SS, primary Sjogren's (sicca) syndrome; trpE, tryptophanyl E. 
$\mathrm{H}_{2} \mathrm{O}$ at $120^{\circ} \mathrm{C}$ for 30 min to denature proteins (28). As described previously, some autoantigens lost reactivity, whereas others retained antibody recognition after this procedure (29). Nitrocellulose transfers were blocked with $3 \%$ skim milk powder and sequentially probed with patient sera (diluted 1:200) and alkaline phosphatase-conjugated goat anti-human IgG (diluted 1:1,000). Blots were scored as positive when the signal given by the test serum with the fusion protein was signifcantly stronger than the signal obtained with a control lysate expressing trpE alone. TrpE and trpE-La fusion peptides were purified from $12 \%$ polyacrylamide gels by electrophoretic elution (30). Two National Institutes of Health (NIH)/Swiss mice were hyperimmunized with the gel-purified trpE protein. Protein concentrations were measured by the method of Bradford (31).

DNA sequencing. Synthetic oligonucleotides (18 mers) complementary to the coding and noncoding strands of the pATH plasmids adjacent to the polylinker sites were synthesized on a 380B DNA synthesizer (Applied Biosystems Inc., Foster City, CA). La inserts were sequenced by primer extension and dideoxy chain termination (32). La SE was subcloned into pGEM 3 (Promega Biotech, Madison, WI) and sequenced by the same methods using SP6 and T7 polymerase primers.

ELISAs. ELISA wells were coated with gel-purified fusion proteins $(2 \mu \mathrm{g} / \mathrm{ml})$ at $4^{\circ} \mathrm{C}$ overnight. The plates were blocked with $1 \%$ BSA and sequentially incubated with patient serum (diluted 1:200) and alkaline phosphatase-conjugated goat anti-human IgG (diluted 1:1,000; Sigma Chemical Co., St. Louis, MO). Plates were developed with p-nitrophenylphosphate and the OD read at $405 \mathrm{~nm}$ on a Dynatech multiscanner (Flow Laboratories, Inc., McLean, VA). Anti-light chain ELISAs were performed in the same way except that goat anti-kappa and anti-lambda reagents (Tago Inc., Burlingame, CA) were added at dilutions of 1:3,000 and were detected with an alkaline phosphataseconjugated rabbit anti-goat IgG. The anti-light chain dilutions were chosen by checkerboard titration of the antibodies against serial dilutions of whole IgG as described in detail elsewhere (33), so that for pooled normal human IgG they produced equal OD values $(\kappa / \lambda$ ratio $=1$ ). The anti-light chain reagents were shown to be specific in that each bound only to the appropriate purified Bence Jones kappa or lambda light chains on dot blots.

One- and two-dimensional isoelectric focusing (IEF) of anti-La antibodies. IgG fractions were purified from patients' sera by ammonium sulfate precipitation and DEAE cellulose chromatography. IgG was labeled with ${ }^{125} \mathrm{I}$ with chloramine $\mathrm{T}$ to a sp act of $1 \mu \mathrm{Ci} / \mu \mathrm{g}$. Radiolabeled IgG anti-La antibodies were then affinity purified from $\mathrm{La}$ peptides on nitrocellulose paper as described (34). Briefly, nitrocellulose transfers were incubated with radiolabeled IgG, extensively washed, and then eluted with freshly deionized $8 \mathrm{M}$ urea/1\% mercaptoethanol/0.05\% Triton X-100/20 $\mu \mathrm{g}$ BSA/10 mM phosphate buffer, pH 4 at room temperature. Approximately $30,000 \mathrm{cpm}$ of affinity purified antibodies were applied to ultrathin IEF agarose gels containing $8 \mathrm{M}$ urea (35), $0.05 \%$ Triton $\mathrm{X}-100$, as well as ampholytes $5-8$ (LKB Instruments, Inc., Gaithersburg, MD), 8-9.5 (LKB Instruments, Inc.), and 2-11 (Serva Biochemicals, Westbury, NY) in a 2:1:2 ratio. After IEF the gels were either autoradiographed or subjected to SDSPAGE in the second dimension (13) and then autoradiographed. To ensure that the results could be compared between samples and between runs, the following proteins were included as internal markers in each lane: BSA, pI 4.9; bovine carbonic anhydrase, pI 5.9; and ribonuclease, pI 9.4). In some experiments nonradiolabeled affinity purified anti-La antibodies were run on one-dimensional agarose IEF gels, transferred to nitrocellulose paper (36), and probed with anti-light chain antibodies.

\section{Results}

Epitope mapping. To identify immunoreactive La peptides, the La cDNA was restricted with various enzymes and the resulting DNA fragments subcloned into the pATH or pUEX expression vectors so that the translational reading frame of each La peptide was maintained. A map of the $13 \mathrm{La}$ subclones produced is presented in Fig. 1. To ensure that the correct DNA fragments had been subcloned and were in the correct reading frame, the DNA inserts were sequenced directly in the pATH vectors or, after subcloning, in pGEM 3 . In all cases at least 50 bases were sequenced from both the $5^{\prime}$ and $3^{\prime}$ ends of the insert, and the coding sequences were confirmed to be correct (with the exception of an A rather than a $\mathrm{T}$ at nucleotide 863 , as also noted by other investigators $[37,38]$ ). To confirm that the levels of expression of each fusion protein were similar and that a La peptide of the appropriate size was expressed, the molecular weight of each fusion protein was determined by Coomassie blue staining of SDS-polyacrylamide gels, and was subjected to immunoblotting with a mouse anti-trpE serum. All peptides with the exception of the total La (predicted fusion protein 78,000; observed 66,000)

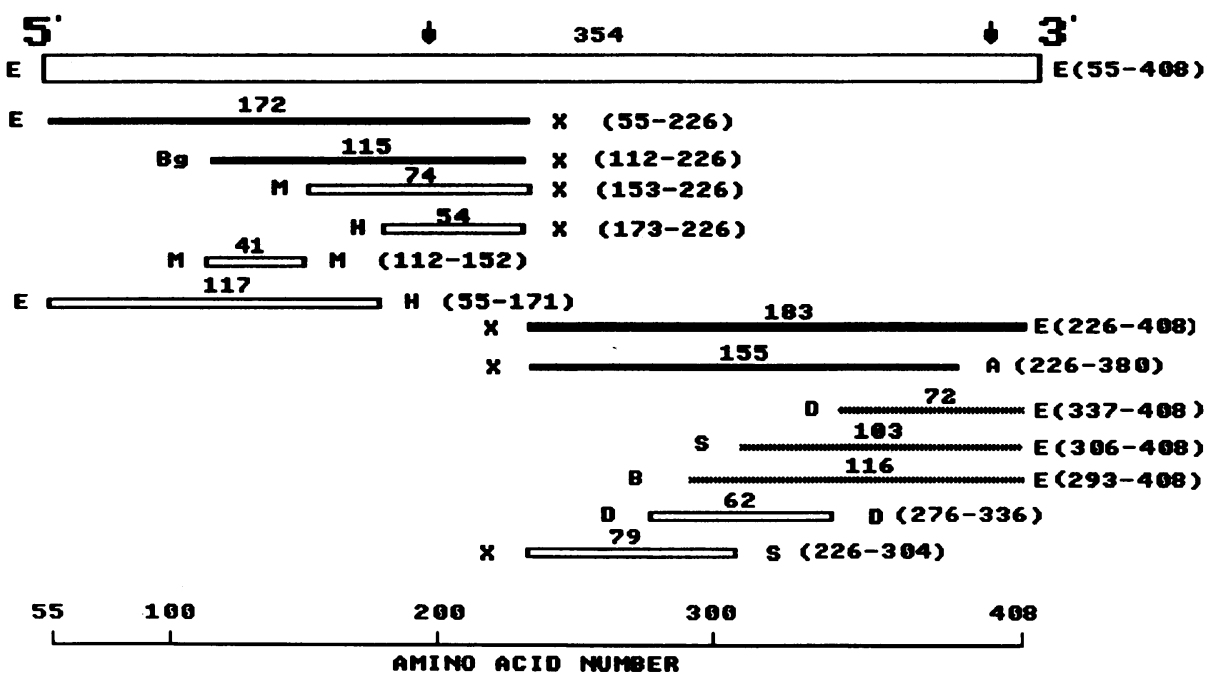
are indicated by arrows. Restriction enzyme key: $E$, Eco RI (synthetic linkers); $B g$, Bgl I; $M$, Mbo I; $H$, Hae III; $A$, Ava II; $D$, Dra I; $B$, BstE II; $S$, Sca I. The cDNA, E-E, encodes 354 of the total 408 amino acids (amino acids 55-408) $(37,38)$
Figure 1. Subclones of La cDNA expressed as peptides. In accordance with the convention used for mapping virus DNA fragments, each subclone is labeled according to the restriction enzyme used for subcloning. Xba I (X) was initially used to restrict the $\mathrm{La}$ cDNA into the $5^{\prime}(\mathrm{EX})$ and $3^{\prime}$ (XE) fragments. The number of amino acids encoded by each subclone is indicated above the subclone and the amino acid number (according to that predicted by full-length human La cDNAs [37, 38]) is indicated in parentheses to the right of each subclone. The shading of horizontal bars reflects the frequency of recognition by anti-La sera: solid, $>70 \%$; stippled, 50-70\%; open, $<10 \%$ (see also Figs. 3 and 5). The sites of the two most hydrophilic portions of $\mathrm{La}$ 


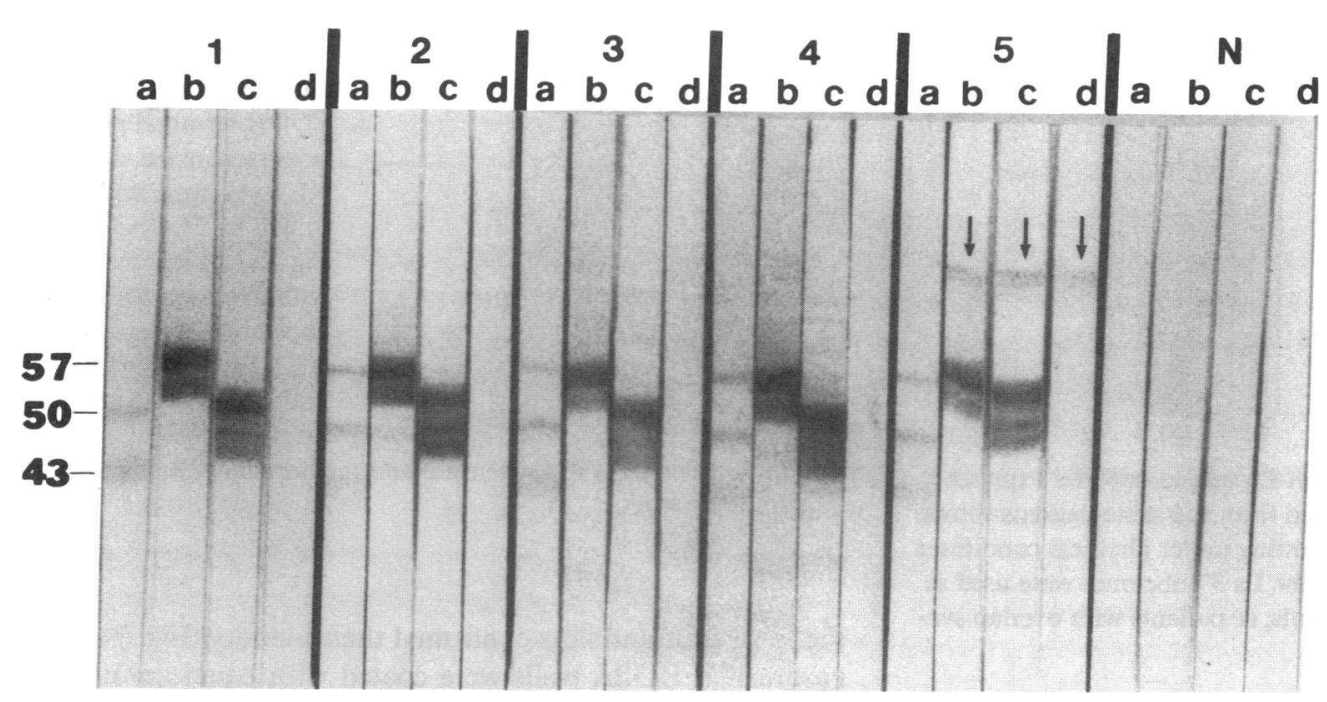

Figure 2. Immunoblot of cell lysates. Extracts from dog spleen (lanes $a$ ) and $E$. coli induced to express La peptides EX-trpE (lanes $b$ ), XE-trpE (lanes $c$ ), or trpE alone (lane $d$ ) were loaded ( $20 \mu \mathrm{g}$ each) on adjacent wells of a $10 \%$ polyacrylamide-SDS gel. After transfer to nitrocellulose paper the strips were probed with a 1:200 dilution of sera derived from either $\operatorname{SLE}(1,2)$, SS $(3-5)$, or a normal control $(N)$. The position of molecular weight markers (pyruvate kinase 57,000 , IgG heavy chain 50,000, and ovalbumin 43,000 ) are shown. The arrows denote binding of serum 5 to $E$. coli proteins in lanes $b-d$.

and XE (predicted fusion protein 58,000; observed 51,000) had a molecular weight within 5,000 of the predicted molecular weight determined by the amino acid sequence. The levels of expression of all fusion proteins used in this study were similar. For reasons that are not understood, the subclone SE was not expressed in the pATH vector but was expressed in the plasmid pUEX.

Initially, La cDNA EE was digested with Xba I, resulting in two DNA fragments encoding peptides of almost equal size $(E X=172$ amino acids and $X E=183$ amino acids; Fig. 1$)$. All patients' sera bound strongly to both of these peptides (Fig. 2, lanes $b$ and $c$ ), whereas normal control sera did not. This reaction was specific for La epitopes since no binding to trpE alone was observed (Fig. 2, lanes $d$ ). As shown previously (3), some SLE and some normal sera bound to $E$. coli proteins on immunoblots, but these were present in all bacterial extracts (Fig. 2 , part 5 , lanes $b-d$ ). Since anti-La sera bound strongly to two nonoverlapping halves of the La protein, this suggested the presence of at least one dominant epitope located on each of the large peptides encoded by EX and XE. To determine whether antigenic sites could be identified on smaller peptides, a further five peptides were derived from EX and a further six from XE. In the case of the La $5^{\prime}$ subclones (Fig. 3), the major epitope was localized to a 115 amino acid Bgl II-Xba encoded fragment $(\mathrm{BgX})$. Virtually all sera reacted as intensely to this peptide as to the whole EX peptide on immunoblots. To verify

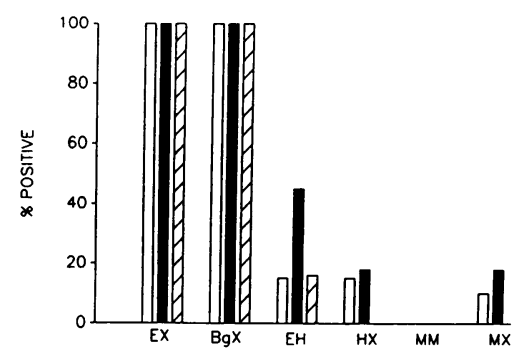

Figure 3. Frequency of binding of anti-La sera to peptides expressed by La $5^{\prime}$ subclones. Sera were obtained from 13 SLE patients (ם), 11 SS patients ( $($ ), and 6 patients with overlap syndromes ( $(x)$ and analyzed by immunoblotting. Immunoblotting was performed as in Fig. 2 except that the peptides encoded by the La $5^{\prime}$ subclones were used as antigens. Antipeptide reactivity was scored as positive when reactivity with the fusion protein was signifcantly greater than reactivity with trpE alone. that the dominant epitope(s) was located within the $\mathrm{BgX}$ fragment, 26 anti-La sera were preincubated with a bacterial lysate containing either the $\mathrm{BgX}$ encoded fusion protein or a lysate containing trpE alone. Sera were absorbed at $4^{\circ} \mathrm{C}$ overnight and tested by the standard ELISA (described below) using EX as the test antigen. At a dilution of 1:200, 19 of $26(73 \%)$ sera preincubated with the $\mathrm{BgX}$ extract showed $>70 \%$ inhibition of binding when compared with sera incubated with lysate containing trpE alone.

Further restriction of the subclone $\mathrm{BgX}$ with Mbo I produced La peptides (MM and MX) with little or no immunoreactivity (Fig. 3). Loss of the major epitope was not due to loss of an epitope encoded at the Mbo I restriction site since an overlapping subclone (EH; Fig. 1) also encoded a peptide with significantly lower immunoreactivity than the EX encoded peptide. These findings suggested that the conformation of the EX-encoded peptide was important for antibody recognition. To test this hypothesis, nitrocellulose strips were probed with anti-La sera after being autoclaved. Anti-La sera failed to bind to $\mathrm{EX}$ (Fig. 4, $A C$, lanes $b$ ) and $\mathrm{BgX}$ (not shown) after denaturation, whereas the same sera still recognized epitope(s) on XE $(A C$, lanes $a$ ). This result could not be explained by differential loss of EX from the nitrocellulose, since immunoblots probed with the anti-trpE serum revealed that both fusion proteins were retained on the nitrocellulose (not shown).

A greater degree of heterogeneity in epitope recognition was observed with anti-La sera binding to peptides encoded by subclones of La XE (Fig. 5). Approximately half of all sera tested bound to the carboxyl- $(\mathrm{COOH}-)$ terminal peptides $\mathrm{DE}$,

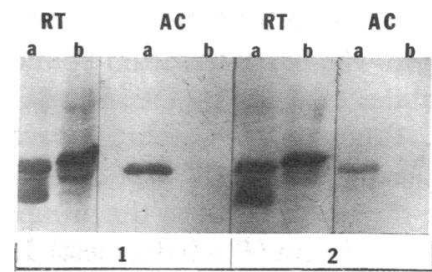

Figure 4. Effect of heat denaturation on EX and XE encoded peptides. Bacterial lysates containing either $\mathrm{XE}$ (lanes $a$ ) or EX (lanes $b$ ) fusion proteins were resolved on by SDS-PAGE, blotted to nitrocellulose, and either left in water at room temperature $(R T)$ or autoclaved $(A C)$. Each pair of strips $(R T$ and $A C)$ was then blocked and probed with a different anti-La containing serum ( 1 and 2). 


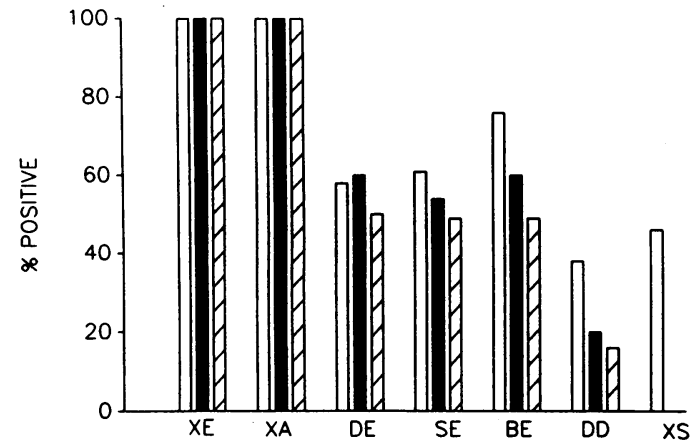

Figure 5. Frequency of binding of anti-La sera to peptides expressed by $\mathrm{La} 3^{\prime}$ subclones. Sera were obtained from the same patients shown in Fig. 3 and analyzed by immunoblotting under identical conditions except that the peptides encoded by the $\mathrm{La} 3^{\prime}$ subclones were used as antigens. $\square$, SLE patients; $\backsim$, SS patients; $\approx$, patients with overlap syndromes.

$\mathrm{SE}$, and $\mathrm{BE}$, although with considerably lower intensity than to the XE encoded peptide. Since most of these sera showed similar binding to the 72 amino acid peptide (encoded by DE) and the 116 amino acid peptide (encoded by $\mathrm{BE}$ ), at least one epitope recognized by half of the patient sera must be located within the COOH-terminal 72 amino acid residues. A hydrophilicity plot (39) revealed a predicted antigenic site located between two prolines (residues 382-393) that contained nine charged amino acids (Fig. 1, second arrow). Surprisingly, however, a peptide lacking the $\mathrm{COOH}$-terminal 28 residues (XA, Fig. 1) showed similar signal intensity on immunoblots as the total La XE peptide (not shown). Together with the lower frequency and intensity of reactivity of anti-La sera with the $\mathrm{DE}$ encoded peptide, this indicates that the $\mathrm{COOH}$ terminus is not immunodominant for any of the sera used in this study.

To determine whether the XA encoded peptide contained all of the epitope(s) recognized by anti-XE, an absorption experiment similar to that described for $\mathrm{BgX}$ was performed with $\mathrm{XA}$ as the competing antigen and $\mathrm{XE}$ as the test antigen. Absorption removed only $38.5 \pm 19.8 \%$ of binding. Attempts were also made to identify epitope(s) in the proximal region of $\mathrm{La} 3^{\prime}$, but only a minority of sera bound to peptides encoded by XS or DD (Fig. 5) and the binding was weak (not shown). Overall, these results indicate that there are at least two epitopes contained within the nonoverlapping peptides encoded by XS and $D E$. Since evidence for a linear epitope in the peptide encoded by XE was obtained by autoclaving (Fig. 4), the weaker reactivity of XS and DE encoded peptides relative to the total $\mathrm{La}$ (XE) peptide suggest that both linear and conformational epitopes are located in this peptide.

Finally, to determine the relationship between antibody titer and the number of peptides in the carboxyl half of $\mathrm{La}$ recognized by the 30 anti-La sera, anti-XE ELISA levels (see below) were grouped according to whether each serum bound to $0-1,2-3$, or $4-5$ of the peptides encoded by DE, SE, BE, $\mathrm{DD}$, and XS on immunoblots. Although a clear trend toward higher anti-XE levels with increasing numbers of peptides recognized was observed (Fig. 6), the levels between the groups were not statistically significantly different $(P>0.05, t$ test $)$. In addition, occasional sera that did not bind to any of the smaller peptides had moderately high anti-XE levels (Fig. 6).

Anti-La ELISAs. A Coomassie blue-stained gel of the SDS-PAGE purified trpE-La EX and XE peptides is shown in

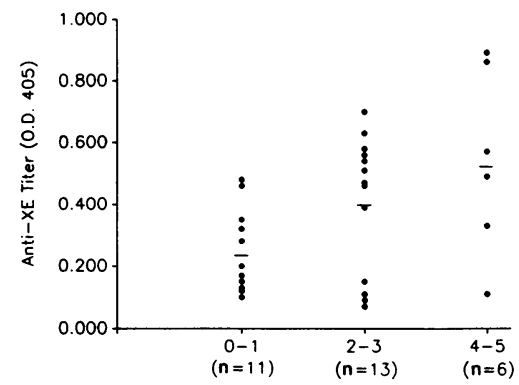

No. XE-Derived Peptides Bound
Figure 6. Relationship between anti-XE levels and the number of COOH-terminal peptides recognized. Anti$\mathrm{XE}$ levels were determined by ELISA (see Fig. 8) and reactivity with five peptides encoded by subclones of $\mathrm{XE}$ (DE, SE, BE, DD, and $X S$ ) analyzed by immunoblotting. $n$ is the number of patients in each group and the horizontal bar is the mean antibody level.

Fig. $7 \mathrm{~A}$. Immunoblots confirmed their identity (Fig. $7 \mathrm{~B}$ ). To ensure that ELISA wells were coated with equal amounts of these fusion proteins, serial dilutions of a mouse anti-trpE serum was used. At the same coating protein concentration (2 $\mu \mathrm{g} / \mathrm{ml})$, the anti-trpE serum reacted equally to the EX and XE fusion proteins used as antigens in the ELISA. When anti-La sera were tested for reactivity against these fusion proteins, the levels of anti-EX $(0.57 \pm 0.23)$ were higher than levels of antiXE (0.23 \pm 0.18$)$. Antibody levels to the two peptides were highly significantly correlated ( $r=0.68, P<0.001$; Fig. 8).

Antibody heterogeneity. To determine the degree of heterogeneity of anti-La antibodies reactive with either the EX or XE peptides, the light chain distribution and spectrotypes of the antibodies were examined. As shown in Fig. 9, a preference for $\kappa$-chain utilization was observed for anti-5' antibodies, whereas anti-3' showed a slight increase in $\lambda$-chain utilization. If $\alpha / \lambda$ ratios $<0.8$ are arbitrarily taken as a $\lambda$ predominance and ratios $>1.2$ are taken as a $\kappa$ predominance, then a $\kappa$ predominance was observed in $20(69 \%)$ anti-La EX but only $2(7 \%)$ anti-XE antibodies. Conversely, $10(34 \%)$ anti-XE, but only 1 (3\%) anti-EX antibodies preferentially used $\lambda$-chains. No major differences were observed in the light chain distribution amongst SLE versus SS patients.

Preliminary attempts at elution of radiolabeled anti-La peptide antibodies from nitrocellulose transfers with glycine $\mathrm{HCl}, \mathrm{pH} 2.6$, were only partially successful since $30-50 \%$ of presumably high affinity antibodies remained bound to antigen on the nitrocellulose. In addition, after neutralization the isoelectric focusing was influenced by the presence of antigen/ antibody complexes (not shown). Elution with $8 \mathrm{M}$ urea in the presence of mercaptoethanol at acid $\mathrm{pH}$ increased antibody recovery to $>80 \%$, maintained the dissociation of antibody from antigens, and minimized or avoided carbamylation (40).

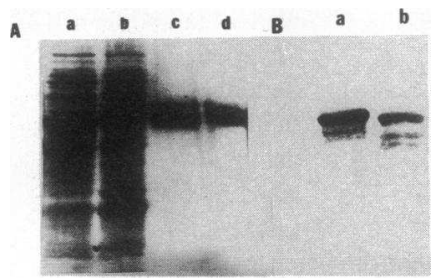

Figure 7. Purified fusion proteins used for ELISA. A, Coomassie blue stain of a polyacrylamide-SDS gel showing the total bacterial extracts (lanes $a-b$ ) containing the overexpressed recombinant fusion proteins and the corresponding electroeluted trpE-XE (lane $c$ ) and trpE-EX (lane $d$ ) fusion proteins. $B$, Immunoblot of the electroeluted fusion proteins using an anti-La serum. Lane $a$, EX-trpE; lane $b$, XE-trpE. 


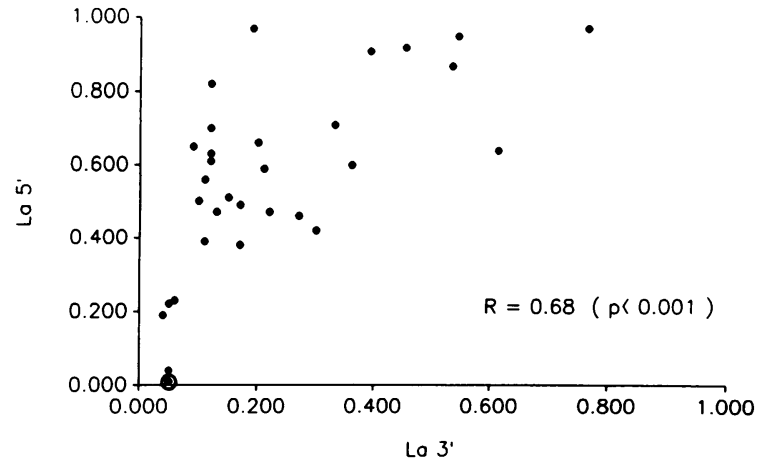

Figure 8. Correlation between anti-EX (La $\left.5^{\prime}\right)$ and anti-XE (La $\left.3^{\prime}\right)$ titers. The purified fusion proteins as shown in Fig. 7 were used to coat ELISA plates at a concentration of $2 \mu \mathrm{g} / \mathrm{ml}$. The plates were blocked with BSA and sequentially incubated with the 30 anti-La sera (1:200 dilution) and alkaline phosphatase-conjugated goat antihuman IgG (1:1,000 dilution). The results are expressed as the $O D$ at $405 \mathrm{~nm}$. The circled dot represents 10 normal sera.

The spectrotypes of ${ }^{125}$ I-labeled affinity purified anti-EX and $X E$ antibodies are shown in Figs. 10 and 11. As shown for two different patients' IgG, the antibody populations had clearly different spectrotypes (Fig. 10, lanes 3-6) and were restricted when compared with a control affinity purified antibody. In addition, oligoclonal bands were observed in the anti-EX antibody population (Fig. 10, lanes 4 and 6). Oligoclonal bands were observed in IgG anti-EX from three of four SS sera tested. Since these bands occurred in the acidic portion of the gels run under reducing conditions, the bands were thought to be light chains. Two-dimensional gel electrophoresis (Fig. 11) confirmed that the spectrotype of the light chains from anti-EX IgG was highly restricted $(D)$ and more similar to that observed for a monoclonal $(B)$ than an affinity-purified polyclonal IgG preparation $(A)$. To identify the oligoclonal bands, nonradiolabeled affinity purified IgG was subjected to IEF, transferred to nitrocellulose paper, and probed with antilight chain antibodies. In two of two preparations studied, the oligoclonal bands were $\kappa$ light chains (not shown).

\section{Discussion}

We have previously shown that anti-La sera bind to peptide fragments of $27 \mathrm{kD}$ and smaller after partial proteolysis (41).
Using radiolabeled methionine and phosphate, Chan et al. demonstrated that the $28-\mathrm{kD}$ fragment $(\mathrm{X})$ contained all of the methionine residues and a $23-\mathrm{kD}$ fragment $(\mathrm{Y})$ contained all of the phosphorylated amino acids (42). Most anti-La sera ( $\sim 70 \%$ in their study) reacted with both peptides. Fortuitously, the two major peptides produced in the present study (encoded by EX and XE) are almost certainly very similar to the $X$ and $Y$ peptides (Fig. 12) except that the peptide encoded by EX lacks the $\mathrm{NH}_{2}$-terminal 54 amino acids of the authentic La protein $(37,38)$. All of the ATG codons for methionine are located upstream of the Xba I restriction site (Fig. 1) and the predicted molecular weights of peptides expressed from Xba I restricted total $\mathrm{La}$ cDNA would be $26,300\left(\mathrm{NH}_{2}\right.$ terminal) and 20,500 (COOH terminal). Using the recombinant DNA approach, we have therefore confirmed that anti-La sera recognize at least two independent epitopes, one located in the $\mathrm{NH}_{2}$-terminal and the other in the $\mathrm{COOH}$-terminal half of the La protein. Further restriction of the EX DNA revealed that the immunodominant epitope resided within a 115 amino acid peptide encoded by $\mathrm{BgX}$. Two observations suggested that the epitope encoded by $\mathrm{BgX}$ is conformational in nature. All subclones of $\mathrm{BgX}$ as well as overlapping cDNA fragments encoded peptides that were either weakly antigenic or not antigenic at all. Secondly, heat denaturation of peptides encoded by $\mathrm{EX}$ or BgX but not XE (or several other autoantigens [29]) caused total loss of recognition by anti-La sera. The peptide encoded by $\mathrm{BgX}$ is similar to the $\mathrm{C}$ peptide (9) and peptide 6 (10) reported by other investigators (Fig. 12). The high frequency of binding of anti-La sera to the C peptide (94\% [9]) is therefore similar to the observations in this study, whereas the total failure of anti-La sera to recognize peptide 6 on immunoblots (10) is difficult to explain. Possibilities include proteolytic degradation or an abnormal folding of $\mathrm{La}$ as part of a cro-B-galactosidase fusion protein.

The smallest peptide recognized by all anti-La sera tested on the COOH-terminal half of the La protein was a 155 residue peptide encoded by the subclone XA. This finding is surprising since the most hydrophilic region and predicted antigenic site on the $\mathrm{COOH}$-terminal half of $\mathrm{La}$ is located within the terminal 26 residues that are lacking in the XA encoded peptide (Fig. 1). The absence of a dominant linear epitope in the COOH-terminal 28 amino acids of $\mathrm{La}$ was confirmed by the absent or weak binding of all anti-La sera tested to a synthetic peptide corresponding to the $\mathrm{COOH}$-terminal 28 residues of La (kindly provided by Dr. N. Brot, Roche Institute of Molecular Biology; unpublished observations). As in the case
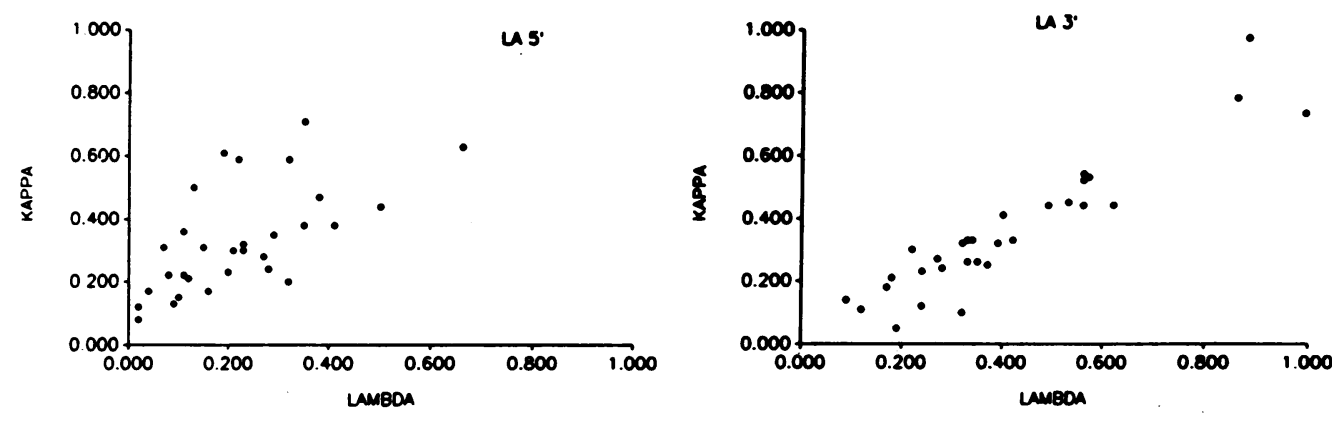

Figure 9. Light chain distribution of anti-La sera as determined by ELISA. The electroeluted EX (La $\left.5^{\prime}\right)$ - or XE (La $3^{\prime}$ )-trpE fusion proteins were used as antigens and sequentially incubated with anti-La sera (1:200 dilution), goat anti-human $\kappa$ or anti- $\lambda$ chains $(1: 3,000$ dilution) and alkaline phosphatase-conjugated rabbit antigoat IgG (1:1,000 dilution). The results are expressed as the OD at $405 \mathrm{~nm}$. 


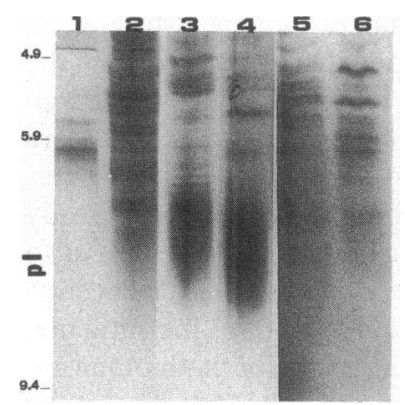

Figure 10. Spectrotypic analysis of anti-La peptide antibodies. IgG was isolated from two SS patients' sera, ${ }^{125}$ I-labeled, and affinity purified from nitrocellulose-bound $\mathrm{EX}$ (lanes 4 and 6) or XE (lanes 3 and 5). The IgG antibodies were then dissociated into heavy and light chains by mercaptoethanol and $30,000 \mathrm{cpm}$. subjected to IEF on ultrathin agarose gels. The spectrotypes of a radiolabeled human IgGk monoclonal antibody (myeloma) (lane 1 ) and a polyclonal IgG affinity purified goat anti-human IgG (Fc fragment) (lane 2) are shown for comparison. The positions of the pI markers, BSA (4.9), bovine carbonic anhydrase (5.9), and RNAse (9.4) are indicated. The gel was developed by autoradiography.

of BgX, further restriction of cDNA encoding a large immunodominant peptide resulted in a significant decrease in both the intensity and frequency of binding to the smaller constituent peptides. However, several features of the epitopes within the $\mathrm{COOH}$-terminal La peptide were different from $\mathrm{BgX}$. Absorption of anti-La sera with the peptide encoded by XA only removed $\sim 40 \%$ of binding to the XE encoded peptide, indicating that although no independent epitope could be identified on the $\mathrm{COOH}$-terminal 28 amino acids, these residues must allow the XE encoded peptide to adopt an optimal conformation or assembled epitope that is recognized by anti-La antibodies. Although conformation was important for binding of anti-La sera to the XE and XA encoded peptides, heat denaturation of these peptides did not completely destroy antibody recognition, suggesting that a linear epitope is located between residues 226 and 380 . Finally, greater variation in binding of anti-La sera to different peptides was observed in the $\mathrm{COOH}$-terminal half of the La protein. Approximately half of the sera reacted with epitope(s) located within the $\mathrm{COOH}$ terminal 72 residues and $20-30 \%$ of sera bound to the more proximal short peptides encoded by DD and XS. All published studies so far have identified at least one epitope in the $\mathrm{COOH}$-terminal half of $\mathrm{La}(7,9,10)$. Our subclones $\mathrm{SE}$ and $\mathrm{BE}$ are very similar to the subclones III (7) and $9(10)$ reported to be strongly antigenic by others (Fig. 12). While we also observed that most anti-La sera recognized peptides encoded by these cDNA fragments, the intensity of binding was usually much less than that observed for the larger peptides encoded by XE and XA.

The shortest independent immunodominant (defined arbitrarily as intense immunoblot reactivity by $70 \%$ or more of sera tested) epitopes identified in this study were encoded by $\mathrm{BgX}$ in the $\mathrm{NH}_{2}$-terminal half of $\mathrm{La}$ and by $\mathrm{XA}$ in the $\mathrm{COOH}$ terminal half of $\mathrm{La}$. As with previous epitope mapping studies of $\mathrm{La}$ and other autoantigens (7-10), antibody binding to small nonoverlapping peptides (EH, HX, DD, and DE) could be taken as evidence for recognition of four separate epitopes. However, we interpret the significantly lower frequency and intensity of antibody binding to these smaller peptides as indicating that the peptides form part of the assembled immunodominant epitopes mentioned above. The trend toward increasing anti-EX titers in sera binding to larger numbers of peptides is compatible with this idea, although some of the heterogeneity in epitope recognition is clearly not simply a function of antibody titer.

In addition to differences in interpretation, we also observed some differences in the location of epitopes on the $\mathrm{La}$ protein and frequency with which they were recognized compared with previous studies $(7,9,10)$. Whereas some of these
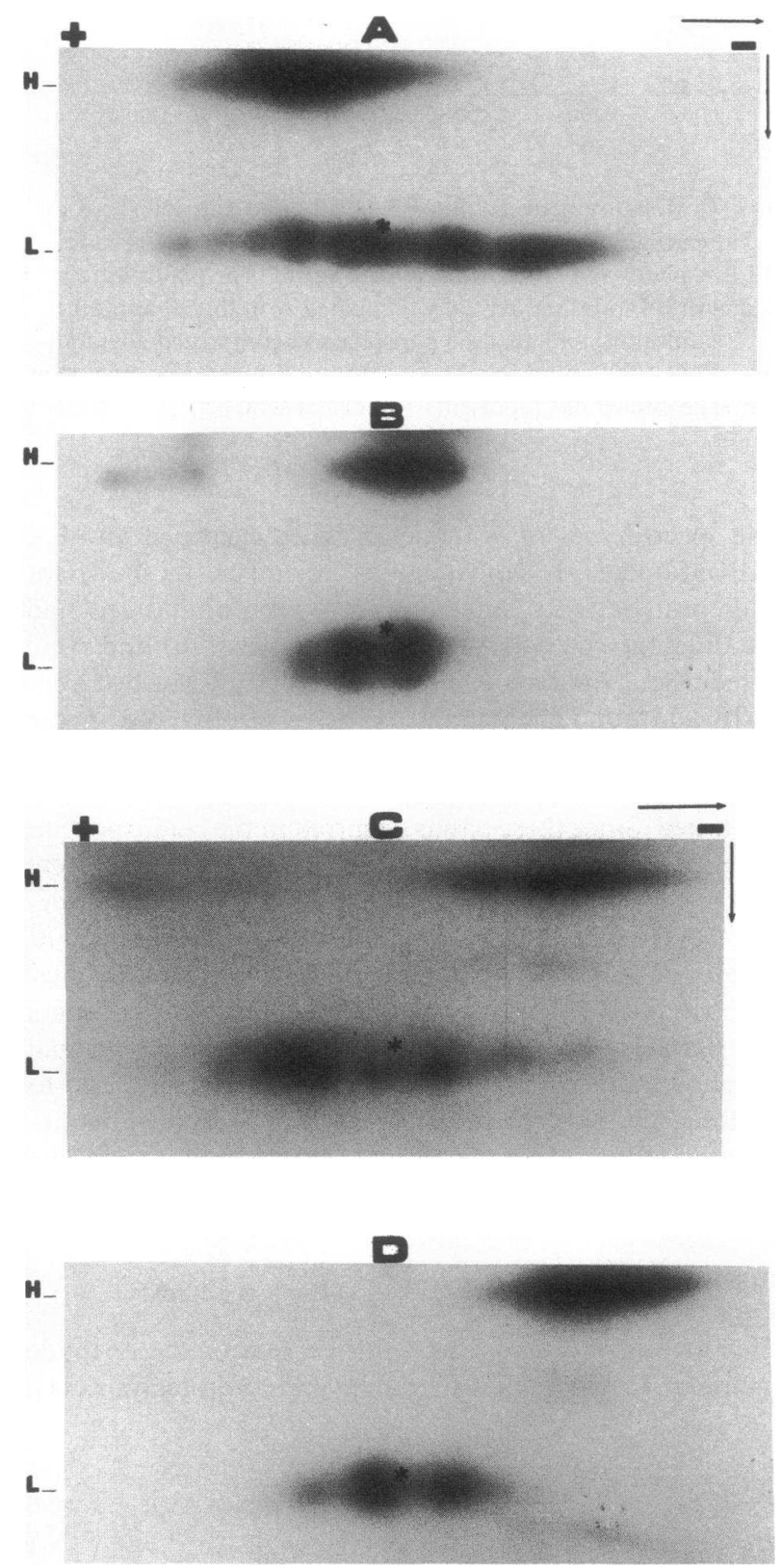

Figure 11. Two-dimensional gel electrophoretic analysis of anti-La peptide antibodies. Radiolabeled, affinity purified anti-La EX $(D)$ and anti-La XE $(C)$ (from the same patient's IgG analyzed in lanes 3 and 4 in Fig. 10), polyclonal $\mathrm{IgG}$ goat anti-human $\mathrm{Fc}(A)$ and a human IgGk myeloma protein $(B)$ were focused in the first dimension (horizontal arrow) as in Fig. 10. The agarose strips were then overlayed on top of $10 \%$ polyacrylamide SDS gels and the heavy $(H)$ and light $(L)$ chains resolved by electrophoresis in the second dimension (vertical arrow). The direction of migration for each dimension, the positions of the anode $(+)$ and cathode $(-)$ for IEF, and the positions of an internal marker, bovine carbonic anhydrase (asterisks), are shown. The gels were developed by autoradiography. 


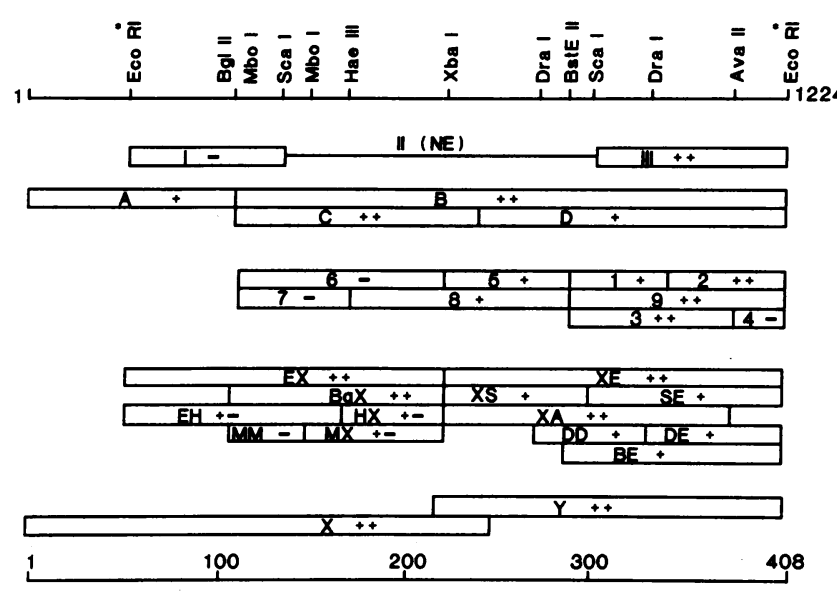

Figure 12. Composite epitope map of the La protein. From top to bottom the map shows the restriction enzymes used for mapping (Eco RI* is a synthetic linker), reactivity of anti-La sera with La peptides (I-III, reference 7; A-D, reference 9; 1-9, reference 10; restriction enzyme-labeled, current study, and $X-Y$, reference 42 ), and the amino acid number. Peptides reported as strongly antigenic $(++)$, positive $(+)$, not antigenic $(-)$, and not expressed $(N E)$ are indicated. All peptides other than $\mathrm{X}$ and $\mathrm{Y}$ (proteolytic cleavage products) were derived from cDNAs.

differences may be explained by the use of small numbers or pools of patient sera in some prior studies, others may be due to technical differences. As mentioned above, variable conformations of the antigenic peptide could arise from fusion to different bacterial proteins as well as by disruption of the normal folding of the protein depending on where the peptide was cleaved. In contrast to partial proteolysis where the enzymes may cut between domains and produce only modest changes in peptide conformation (43-45), the sites of cDNA digestion (and hence peptide cleavage) are dictated by the convenience of DNA restriction sites. A third possible explanation for some differences observed in this study is the use of trpE rather than B-galactosidase to form a fusion protein. Proteolytic cleavage of a peptide antigen is difficult to detect on B galactosidase, both because of its high molecular mass $(120 \mathrm{kD})$ and its own susceptibility to proteolytic breakdown $(7,9)$. In contrast, we observed little degradation of $\operatorname{trpE}$ in protein lysates, and the proteolytic cleavage observed for the large peptides encoded by EX and XE do not effect the interpretations of this study.

It has been suggested that any exposed portion of a protein may be recognized as a $B$ cell antigen depending on the nature of the protein and a variety of host factors (46). With the exception of the peptide encoded by XS, we observed striking similarities in the peptide binding patterns of anti-La sera in SLE, SS, and overlap syndromes. The relatively small number of dominant epitopes in an outbred population with different diseases as well as the identification of immunodominant epitopes on other autoantigens (CEN-B [47], insulin in untreated diabetics [48], the acetylcholine receptor [49], the ribosomal $P$ proteins [4]) suggests that autoantibody diversity is constrained (see also below). Although immunodominant epitopes on the P proteins (6) and alpha subunit of the acetylcholine receptor (49) correspond to predicted antigenic sites based on surface orientation and hydrophilicity, the failure of hydrophilicity alone to predict the immunodominant sites on La suggests that other factors related either to the antigen (e.g., tertiary structure, posttranslational modifications, RNA binding) or the autoantibodies (e.g., highly conserved genes encoding autoantibodies) are important. Regardless of the constraints of autoantibody production, the strong correlation observed between the levels of antibodies to the dominant peptides suggests that the La protein itself is stimulating antiLa production in these patients.

The detection of two immunodominant epitopes on nonoverlapping La peptides that do not share amino acid sequence homology implies the existence of multiple anti-La reactive B cell clones in each patient. To verify this and to determine the degree of heterogeneity of the antibodies binding to each immunodominant peptide, we analyzed the $\kappa / \lambda$ ratios and spectrotypes of anti-La EX and anti-La XE antibodies. The differences in the patterns of light chain utilization (predominantly $\kappa$-chain for anti-La EX and either $\lambda$-chain predominance or a normal ratio for anti-La XE) as well as differences in the spectrotypes confirmed that the populations of antibodies binding to each peptide were distinct. These results are in agreement with a previous analysis of antibodies against the total La protein which showed an overall predominance of $\kappa$-chain utilization and a polyclonal antibody response (50). To evaluate antipeptide heterogeneity due to the $V_{H}$ and $V_{L}$ regions of the autoantibodies, IgG was isolated from patients' sera, affinity purified from the La EX and XE peptides, and subjected to IEF. To ensure elution of high affinity antibodies which are frequently retained on immobilized antigens (51), mercaptoethanol and urea were added to the elution buffer. Analysis of the eluted antibodies revealed considerable restrictions in anti-La EX and anti-La XE heterogeneity as well as oligoclonal bands corresponding to $\kappa$ light chains in the small number of samples studied. The limited diversity of the antipeptide heavy and light chains is partly due to the removal of isotypes other than IgG before IEF, the almost exclusive utilization of the IgG1 subclass by anti-La antibodies $(50,52)$, and the presence of urea in agarose IEF gels. Whereas individual antibody clones produce approximately four to six bands in agarose IEF gels in the absence of urea $(36,53)$, an IgG myeloma produced two bands corresponding to the dissociated heavy and light chains in the presence of urea (Fig. 10). Although we cannot be certain that the antibodies analyzed are completely representative of the total IgG response to the native $\mathrm{La}$ protein, the striking restriction in the spectrotypes of antibodies to the two large immunodominant peptides strongly suggests that there is a pauciclonal expansion of anti-La reactive B cells. The antiLa peptide restriction is similar to that observed with the immunodominant epitope on the ribosomal $P$ proteins analyzed by radiolabeled antigen overlay of focused $\mathrm{IgG}(5)$. Whether such restriction is due to a smaller pool of autoreactive B cells or to maturation of the immune response with selection of the highest affinity antibodies (54) cannot be distinguished by the present studies. However, extensive expansion of a relatively limited number of autoreactive B cell clones is in keeping with the detection of paraproteins in the sera and urine of SS patients $(55,56)$, as well as the increased frequency of pseudolymphomas in patients with this disease (57).

\section{Acknowledgments}

This work was supported by grants AR-38915 and a Research Career Development Award (to Dr. Elkon) from the NIH. 


\section{References}

1. Tan, E. M. 1982. Autoantibodies to nuclear antigens (ANA): their immunobiology and medicine. Adv. Immunol. 33:167-240.

2. Christian, C. L., and K. B. Elkon. 1986. Autoantibodies to intracellular proteins: clinical and biological implications. Am. J. Med. 80:53-61.

3. Gharavi, A. E., J. L. Chu, and K. B. Elkon. 1988. Autoantibodies in systemic lupus erythematosus are not due to random polyclonal B cell activation. Arthritis Rheum. 31:1337-1345.

4. Elkon, K. B., S. Skelly, A. Parnassa, W. Moller, W. Danho, H. Weissbach, and N. Brot. 1986. Identification and chemical synthesis of a ribosomal protein antigenic determinant in systemic lupus erythematosus. Proc. Natl. Acad. Sci. USA. 83:7419-7423.

5. Bonfa, E., J. L. Chu, N. Brot, and K. B. Elkon. 1987. Lupus antiribosomal $P$ peptide antibodies show limited heterogeneity and are predominantly of the IgG1 and IgG2 subclasses. Clin. Immunol. Immunopathol. 45:129-138.

6. Elkon, K. B., E. Bonfa, R. Llovet, W. Danho, H. Weissbach, and N. Brot. 1988. The properties of the ribosomal P2 protein autoantigen are similar to those of foreign antigens. Proc. Natl. Acad. Sci. USA. 85:5186-5189.

7. Sturgess, A. D., M. G. Peterson, L. J. McNeilage, S. Whittingham, and R. O. Coppel. 1988. Characteristics and epitope mapping of a cloned human autoantigen La. J. Immunol. 140:3212-3218.

8. Guldner, H. H., H. J. Netter, C. Szostecki, H. J. Lakomek, and H. Will. 1988. Epitope mapping with a recombinant $68-\mathrm{kDa}$ (U1) ribonucleoprotein antigen reveals heterogeneous autoantibody profiles in human autoimmune sera. J. Immunol. 141:469-475.

9. St. Clair, E. W., D. S. Pisetsky, C. F. Reich, and J. D. Keene. 1988. Analysis of autoantibody binding to different regions of the human $\mathrm{La}$ antigen expressed in recombinant fusion proteins. $\mathrm{J}$. Immunol. 141:4173-4180.

10. Rauh, J. G., H. Hornig, and R. Luhrmann. 1988. At least three distinct B cell epitopes reside in the C-terminal half of La protein, as determined by a recombinant DNA approach. Eur. J. Immunol. 18:2049-2057.

11. Akizuki, M., M. J. Boehm-Truitt, S. S. Kassan, A. D. Steinberg, and T. M. Chused. 1977. Purification of an acidic nuclear protein antigen and demonstration of its antibodies in subsets of patients with sicca syndrome. J. Immunol. 119:932-939.

12. Hendrick, J. P., S. L. Wolin, J. Rinke, M. R. Lerner, and J. A Steitz. 1981. Ro small cytoplasmic ribonucleoproteins are a subclass of La ribonucleoproteins: further characterization of the Ro and La small ribonucleoproteins from uninfected mammalian cells. Mol. Cell. Biol. 1:1138-1149.

13. Elkon, K. B., and L. Culhane. 1984. Partial immunochemical characterization of the Ro and La proteins using antibodies from patients with the sicca syndrome and lupus erythematosus. J. Immunol. 132:2350-2356.

14. Francoeur, A. M., and M. B. Mathews. 1982 . The interaction between VA RNA and the lupus antigen La: formation of a ribonucleoprotein particle in vitro. Proc. Natl. Acad. Sci. USA. 79:6772-6776.

15. Rinke, J., and J. A. Steitz. 1982. Precursor molecules of both human 5S ribosomal RNA and transfer RNAs are bound by a cellular protein reactive with anti-La lupus antibodies. Cell. 29:149-159.

16. Gottlieb, E., and J. Steitz. 1989. Function of the mammalian La protein: evidence for its action in transcription termination by RNA polymerase III. EMBO (Eur. Mol. Biol. Organ.) J. 8:851-861.

17. Alspaugh, M. A., N. Talal, and E. M. Tan. 1976. Differentiation and characterization of autoantibodies and their antigens in Sjogren's syndrome. Arthritis Rheum. 19:216-222.

18. Mattioli, M., and M. Reichlin. 1974. Heterogeneity of RNA protein antigen reactive with sera of patients with systemic lupus erythematosus. Arthritis Rheum. 17:421-429.

19. Elkon, K. B., A. E. Gharavi, G. R. V. Hughes, and H. M. Moutsopoulos. 1984. Autoantibodies in the sicca syndrome (primary Sjogren's syndrome). Ann. Rheum. Dis. 43:243-245.
20. Watson, R. M., A. T. Lane, M. K. Barnett, W. B. Bias, F. C. Arnett, and T. T. Provost. 1984. Neonatal lupus erythematosus: a clinical, serological, and immunogenetic study with review of the literature. Medicine (Baltimore). 63:362-378.

21. Silverman, E. D., M. J. Mamula, J. A. Hardin, and R. M. Laxer. 1989. The association of the congenital heart block (CHB) of neonatal lupus erythematosus (NLE) and both anti-Ro and anti-La antibodies. Arthritis Rheum. 32:S104. (Abstr.)

22. Tan, E. M., A. S. Cohen, J. F. Fries, A. T. Masi, D. J. McShane, N. F. Rothfield, J. G. Schaller, N. Talal, and R. J. Winchester. 1982. The 1982 revised criteria for the classification of systemic lupus erythematosus. Arthritis Rheum. 25:1271-1277.

23. Elkon, K. B., A. E. Gharavi, B. M. Patel, G. R. V. Hughes, and A. Frankel. 1983. IgA and IgM rheumatoid factors in serum, saliva, and other secretions: relationship to immunoglobulin ratios in systemic sicca syndrome and rheumatoid arthritis. Clin. Exp. Immunol. 52:75-84.

24. Bressan, G. M., and K. K. Stanley. 1987. pUEX, a bacterial expression vector related to $\mathrm{pEX}$ with universal host specificity. $\mathrm{Nu}$ cleic Acids Res. 15:10056.

25. Kleid, D. G., D. Yansura, B. Small, D. Dowbenko, D. M. Moore, M. J. Grubman, P. D. McKercher, D. O. Morgan, B. H. Robertson, and H. L. Bachrach. 1981. Cloned viral protein vaccine for foot and mouth disease: response in cattle and swine. Science (Wash. DC). 214:1125-1129.

26. Laemmli, U. K. 1970. Cleavage of structural proteins during the assembly of the head of bacteriophage T4. Nature (Lond.). 227:680-685.

27. Towbin, H., T. Staehelin, and J. Gordon. 1979. Electrophoretic transfer of proteins from polyacrylamide gels to nitrocellulose sheets: procedure and some applications. Proc. Natl. Acad. Sci. USA. 76:4350-4355.

28. Swerdlow, P. S., D. Finley, and A. Varshavsky. 1986. Enhancement of immunoblot sensitivity by heating of hydrated filters. Anal. Biochem. 156:147-153.

29. Elkon, K. B. Nitrocellulose transfer techniques for the characterization, cloning and synthesis of autoantigens. Electrophoresis. 88: In press.

30. Stephens, R. E. 1975. High resolution preparative SDS-polyacrylamide gel electrophoresis. Fluorescent visualization and electrophoretic elution: concentration of protein bands. Anal. Biochem. 65:369-379.

31. Bradford, M. M. 1976. A rapid and sensitive method for the quantitation of microgram quantities of protein utilizing the principle of protein-dye binding. Anal. Biochem. 72:248-254.

32. Sanger, F., S. Nickleu, and A. R. Coulson. 1977. DNA sequencing with chain termination inhibitors. Proc, Natl. Acad. Sci. USA. 74:5463-5467.

33. Chu, J. L., A. E. Gharavi, and K. B. Elkon. 1988. Cryoglobulinemia: analysis of isotype, idiotype, and antibody activity by composite gel electrophoresis. Electrophoresis. 9:121-125.

34. Elkon, K. B., A. P. Parnassa, and C. L. Foster. 1985. Lupus autoantibodies target ribosomal P proteins. J. Exp. Med. 162:459-471.

35. Olsson, I., and T. Laas. 1981. Isoelectric focusing in agarose under denaturing conditions. J. Chromatogr. 215:373-378.

36. Elkon, K. B. 1984. Isoelectric focusing of human IgA and secretory proteins using thin layer agarose gels and nitrocellulose capillary blotting. J. Immunol. Methods. 66:313-321.

37. Chambers, J. C., D. Kenan, B. J. Martini, and J. D. Keene. 1988. Genomic structure and amino acid sequence domains of the human La autoantigen. J. Biol. Chem. 263:18043-18051.

38. Chan, E. K. L., K. F. Sullivan, and E. M. Tan. 1989. Ribonucleoprotein SS-B/La belongs to a protein family with consensus for RNA binding. Nucleic Acids Res. 17:2233-2244.

39. Hopp, T. P., and K. R. Woods. 1981. Prediction of protein antigenic determinants from amino acid sequences. Proc. Natl. Acad. Sci. USA. 78:3824-3828.

40. Stark, G. R., W. H. Stein, and S. Moore. 1969. Reactions of the 
cyanate present in aqueous urea with amino acids and proteins. J. Biol. Chem. 235:3177-3181.

41. Elkon, K. B., and P. W. Jankowski. 1985. Fine specificities of autoantibodies directed against the $\mathrm{Ro}, \mathrm{La}, \mathrm{Sm}, \mathrm{RNP}$ and Jo-1 proteins defined by two-dimensional gel electrophoresis and immunoblotting. J. Immunol. 134:3819-3824.

42. Chan, E. K. L., A. M. Francoeur, and E. M. Tan. 1986. Epitopes, structural domains, and asymmetry of amino acid residues in SS-B/La nuclear protein. J. Immunol. 136:3744-3749.

43. Speicher, D. W., J. S. Morrow, W. J. Knowles, and V. T. Marchesi. 1980. Identification of proteolytically resistant domains of human erythrocyte spectrin. Proc. Natl. Acad. Sci. USA. 77:56735677.

44. Yurchenco, P. D., D. W. Speicher, J. S. Morrow, W. J. Knowles, and V. T. Marchesi. 1982. Monoclonal antibodies as probes of domain structure of the spectrin alpha subunit. J. Biol. Chem. 257:9102-9107.

45. Pierschbacher, M. D., E. G. Hayman, and E. Ruoslahti. 1981. Location of the cell attachment site in fibronectin with monoclonal antibodies and proteolytic fragments of the molecule. Cell. 26:259267.

46. Benjamin, D. C., J. A. Berzofsky, I. J. East, F. R. N. Gurd, C. Hannum, S. J. Leach, E. Margoliash, J. G. Michael, A. Miller, E. M Prager, M. Reichlin, E. E. Sercarz, S. J. Smith Gill, P. E. Todd, and A. C. Wilson. 1984. Annu. Rev. Immunol. 2:67-101.

47. Earnshaw, W. C., P. S. Machlin, B. J. Bordwell, N. F. Rothfield, and D. W. Cleveland. 1987. Analysis of anticentromere autoantibodies using cloned autoantigen CENP-B. Proc. Natl. Acad. Sci. USA. 84:4979-4983.

48. Nell, L. J., C. Hulbert, and J. W. Thomas. 1989. Human insulin autoantibody fine specificity and $\mathrm{H}$ and $\mathrm{L}$ chain use. J. Immunol. 142:3063-3069.

49. Barkas, T., A. Mauron, B. Roth, C. Alliod, S. J. Tzartos, and M. Ballivei. 1987. Mapping of the main immunogenic region and toxin binding site of the nicotinic acetylcholine receptor. Science (Wash. DC). 235:77-80.

50. Yount, W. J., and D. C. Pearce. 1986. Subclass restriction of anti-SS-B (La) autoantibodies. Clin. Immunol. Immunopathol. 38:111-119.

51. Goding, J. W. 1983. Monoclonal Antibodies: Principles and Practice. Academic Press, New York. 188.

52. Bonfa, E., R. Llovet, and K. B. Elkon. 1988. Immunoblot analysis of IgG subclasses of multiple lupus autoantibodies. J. Immunol. 140:2231-2236.

53. Sinclair, D., D. M. V. Parrott, and D. I. Stott. 1986. Quantitation of monoclonal immunoglobulins by isoelectric focusing and its application for monitoring secretory B cell neoplasia. J. Immunol. Methods. 90:247-253.

54. Wysocki, L., T. Manser, and M. L. Gefter. 1986. Somatic evolution of variable region structures during an immune response. Proc. Natl. Acad. Sci. USA. 83:1847-1851.

55. Sugai, S., S. Shimizu, Y. Hirose, T. Tahiguchi, S. Konda, and H. Tamano. 1985. Monoclonal gammopathies in Japanese patients with Sjogren's syndrome. J. Clin. Immunol. 5:90-101.

56. Moutsopoulos, H. M., R. Costello, A. A. Drosos, A. K. Mavridis, and H. M. Papadopoulos. 1985. Demonstration and identification of monoclonal proteins in the urine of patients with Sjogren's syndrome. Ann. Rheum. Dis. 44:109-112.

57. Talal, N., and J. J. Bunim. 1964. The development of malignant lymphoma in the course of Sjogren's syndrome. Am. J. Med. 36:529-540. 